\title{
What now and what next? The new era of OSCE
}

Majid Ali

College of Pharmacy, Umm Al-Qura University, Saudi Arabia \& Faculty of Health and Medical Sciences, University of Adelaide, Australia

\author{
Keywords \\ COVID-19 \\ Pandemic \\ Lockdown \\ Online Assessment \\ OSCE
}

\section{*Corresponding author: maaali@uqu.edu.sa\& majid.ali@hotmail.com}

\begin{abstract}
Summary: The COVID-19 pandemic has impacted all walks of life including the education sector. The suspension of on-campus activities within universities in the last few months, in the middle of a semester, allowed pharmacy educators to capitalise on existing technology in teaching and assessment. Objective structured clinical exams (OSCEs) which usually require a face-to-face assessment of skills, posed a special challenge to educators in the health profession including pharmacy. Several teleconference programmes have been trialed to conduct online or virtual OSCEs. Lack of resources and human workforce compelled faculty to utilise MyDispense, a web-based community pharmacy simulation programme, for conducting web-based OSCEs. The students agreed that this was an overall better learning experience compared to the traditional face-to-face OSCE. There is a need for a robust evaluation of these alternate methods of OSCE in contrast with traditional face-to-face OSCE. The accumulation of more literature is expected to be seen that would highlight the feasibility of the online or virtual OSCE capitalising on advancements in technology. This coupled with the emerging role of telepharmacy, and less complicated skills needing to be assessed in pharmacy education compared to nursing and medication education, could this method of OSCE be the future of pharmacy education?
\end{abstract}

\section{Background and Context}

The world has changed in the last few months as coronavirus spreads across the world making COVID-19, the disease caused by the virus, a pandemic. It is essentially a global health crisis; however, it has impacted all walks of life including the education sector. With the suspension of oncampus activities in the middle of a semester at universities around the world due to the pandemic, educators, as well as learners, were thrown in at the deep end and were forced to adapt to distance learning, taking the form of online education in many instances.

In this unprecedented situation, not only did the teaching methods need to be adjusted, but assessment had to be modified too. Not all faculty and students have the same capability for technology acceptance, however, all have been forced to embrace the existing technology and capitalise on technical skills. 'Were we ready to move online? Did we have sufficient resources? Did we provide appropriate instructions to our students? What impact did it have on the students' learning?'. All these questions are yet to be explored. Perhaps some of the most important aspects of concern are whether modified assessment methods served the purpose and whether faculty were able to craft reliable and valid assessments. This becomes more of a concern in the case where professional skills, communication skills, and other practical skills, in the context of face-to-face situations, are required to be assessed such as in an objective structured clinical examination (OSCE). This commentary will highlight some of the approaches that have been taken recently to conduct the OSCEs in pharmacy education. It will also provide some food for thought for concerned educators regarding how the timely modifications in this assessment method can better prepare pharmacy students for the changing future. 


\section{Educational Description}

On the optimistic side, there has been an abundance of advanced technology at hand in this era, which was and has been able to lessen the pain of the pandemic. Several universities and institutions around the world compiled together the instructions and useful resources to help the academics move to online teaching and assessment. Online teaching and assessment is not a new approach but the current pandemic has revived the need to rely on it. Since some assessment is better than no assessment, we went on a mission to conduct the OSCEs online. Suspension of on-campus activities was surely a hindrance, but faculty were creative and utilised the existing technology resources to meet their needs. Similar to the majority of the medical and nursing colleges, pharmacy colleges also explored the options in the fittest-to-survival race. Numerous teleconferencing programmes such as Zoom, Cisco Webex, and Microsoft Meetings using breakout rooms have been tried to conduct virtual or online OSCEs. Education Management Solutions (EMS) provided a special platform to healthcare education and healthcare providers to accommodate virtual OSCE (EMS, 2020). There has been remarkable feedback from colleagues who have ventured onto this platform. With these online or virtual OSCEs, the human workforce, including the examiners and simulated or standardised patients, were still required but travel time required to the campus or OSCE location was saved to a great extent.

Lack of resources can sometimes push to redefine education. As with asynchronous learning and teaching, faculty endeavoured asynchronous OSCEs, using the simulation leaning system, MyDispense. This was also partly driven by limited financial resources and the human workforce that was available. MyDispense is a free web-based virtual community pharmacy simulation program, originally developed by Monash University (Australia), and has now been widely adopted by many colleges of pharmacy around the world at different levels. It allows educators to create and deliver exercises mimicking real-life case scenarios for students covering a wide range of legal and clinical problems. Faculty already use this programme in a variety of their therapeutics courses and online training, hence students are very familiar with the system. MyDispense was used to conduct web-based OSCEs after creating timed scenarios in it, ensuring not to jeopardise the learning outcomes and pedagogical considerations of the OSCE. Students had a specific time duration to complete their tasks in this webbased OSCE. Students had mixed opinions regarding their experience of using MyDispense for web-based OSCE, although the majority of them agreed that they learned from this method of OSCE more than what they could have from the actual face-to-face OSCE. This could mainly be attributed to the tailored detailed instant feedback which the students received after completing their tasks in this web-based OSCE. For this reason, perhaps, this method can be more rightly called teaching OSCE (TOSCE). The students did not appreciate the aspect that there was no real face-to-face interaction with simulated patients or healthcare professionals. They also thought that they achieved less marks in this web-based OSCE compared to what they could have achieved in the actual face-to-face OSCE. This was an extraordinary learning experience for us. There is much that can be improved regarding the way faculty approached it, however, there is surely a potential for using this OSCE method in the future. More detailed information regarding the process and the results are to be published soon.

Whatever it is called, online OSCE, virtual OSCE (VOSCE), webbased OSCE, teleOSCE, TOSCE, it has been a saviour. However, with tremendous variation in the platforms used, the training and skills of the academic staff, and standardised patients to use the technology; whether it compromised the learning experience of students, or whether sufficient feedback was provided to learners, is remains as yet known and necessitates a standardised framework for alternative OSCE methods.

\section{Outcomes and Recommendations}

The pandemic will hopefully be over but online learning and assessment are here to stay, and perhaps the online or virtual OSCE too. For higher education, it may not be incorrect to say that the pandemic proved to be a blessing in disguise. Faulty incidentally stumbled upon approaches and resources which had not been considered or tried before. While some literature had already started to emerge reflecting on the modified use of OSCEs in nursing and medical education (Boursicot et al., 2020; Lara et al., 2020; Major et al., 2020), the author expects to see good quality literature to support online or virtual OSCEs in pharmacy education from around the world in the near future. There is a great need to evaluate whether modified methods of OSCE compromise the pharmacy education standards, and whether fit-topractice graduates are produced.

As it is said, 'never waste a crisis', the pandemic has provided various opportunities to explore. Telemedicine has not been given the limelight it deserved before this pandemic. There would be a need to include the component of telepharmacy in curricula, as is necessitated by the shift in current practice, and assess students via online or virtual OSCE. Although, online or virtual OSCE has been used before the pandemic to train healthcare students for telemedicine (Sartori et al., 2019), this is a need for educators to embark on preparing students for telemedicine. Teaching the millennial generation of students, more equipped with technology skills every academic year, the use of artificial intelligence in OSCE and also in telepharmacy practice, is also an untapped resource. Who knows whether Amazon Alexa may be being used in OSCE instead of standardised patients or in telepharmacy practice for basic counselling instead of pharmacists, whereby releasing pharmacists for wider clinical responsibilities. With all these advancements, financial resources to adopt new technology will always be a limiting factor, unless affordable resources can be developed. 
Little did Palmer and colleagues know in 2015, when they piloted teleOSCE to train their remote learners in medical education (Palmer et al., 2015), that this could be the next generation OSCE. Since educators in pharmacy education do not require complex patient assessment or medication administration skills such as in medical and nursing education, is this online or virtual OSCE in pharmacy education going to be the future, while maintaining reliability, validity and integrity? Time will tell.

\section{References}

Boursicot, K., Kemp, S., Ong, T.H., Wijaya, L., Goh, S.H., Freeman, K., \& Curran, I. (2020). Conducting a high-stakes OSCE in a COVID-19 environment. MedEdPublish, 9(1). https://doi.org/10.15694/mep. $\underline{2020.000054 .1}$

EMS. (2020). SIMULATIONi Virtual OSCE, Simulation Training Online. Education Management Solutions (EMS). Available at: https:// www.simulationiq.com/virtual-osce

Lara, S., Foster, C.W., Hawks, M., \& Montgomery, M. (2020). Remote Assessment of Clinical Skills during COVID-19: A Virtual, High-Stakes, Summative Pediatric OSCE. Academic Pediatrics, 20(6), 760-761. https://doi.org/10.1016/i.acap.2020.05.029

Major, S., Sawan, L., Vognsen, J., \& Jabre, M. (2020). COVID-19 pandemic prompts the development of a Web-OSCE using Zoom teleconferencing to resume medical students' clinical skills training at Weill Cornell Medicine-Qatar. BMJ Stel. 05/05/2020. https:// doi.org/10.1136/bmjstel-2020-000629

Palmer, R.T., Biagioli, F.E., Mujcic, J., Schneider, B.N., Spires, L., \& Dodson, L.G. (2015). The feasibility and acceptability of administering a telemedicine objective structured clinical exam as a solution for providing equivalent education to remote and rural learners. Rural Remote Health, 15(4), 3399

Sartori, D.J., Olsen, S., Weinshel, E., \& Zabar, S.R. (2019). Preparing trainees for telemedicine: a virtual OSCE pilot. Medical Education, 53(5), 517-518. https://doi.org/10.1111/medu.13851 